\title{
Automation technology as a key component of the Industry 4.0 production development path
}

\author{
Christian Brecher $^{1}$ - Aleksandra Müller ${ }^{1}$ - Yannick Dassen ${ }^{1} \cdot$ Simon Storms ${ }^{1}$
}

Received: 22 December 2020 / Accepted: 4 May 2021 / Published online: 20 May 2021

(C) The Author(s) 2021

\begin{abstract}
Since 2011, the Industry 4.0 initiative is a key research and development direction towards flexible production systems in Germany. The objective of the initiative is to deal with the challenge of an increased production complexity caused by various factors such as increasing global competition between companies, product variety, and individualization to meet customer needs. For this, Industry 4.0 envisions an overarching connection of information technologies with the production process, enabling smart manufacturing. Bringing current production systems to this objective will be a long transformation process, which requires a coherent migration path. The aim of this paper is to represent an exemplary production development way towards Industry 4.0 using eminent formalization approaches and standardized automation technologies.
\end{abstract}

Keywords Industry $4.0 \cdot$ Development path $\cdot$ Automation technology $\cdot$ RAMI 4.0 $\cdot$ Smart manufacturing

\section{Introduction}

Nowadays, industrial production has to deal with the challenge of rapidly increasing global competition between companies from different world regions with various economic conditions. Given the fact that production costs in Europe are generally higher than these in developing countries, European companies are forced to increase product variety and customization to stay competitive [1]. Moreover, fast-changing market conditions cause higher levels of uncertainty and accelerate product life cycles. All these challenges encourage modern manufacturing systems to be highly efficient, adaptable, and eminently flexible, which leads to an increased production complexity [2].

In Germany, the Industry 4.0 initiative is a key research and development direction towards flexible production systems since 2011. This initiative focuses on handling the complexity of production and product life cycles by improving vertical and horizontal integration of production

Aleksandra Müller

aleksandra.mueller@wzl.rwth-aachen.de

1 Laboratory for Machine Tools and Production Engineering (WZL), RWTH Aachen University, Steinbachstraße 19, 52074 Aachen, Germany process participants [3]. To achieve this purpose, a global connection of information technologies with the production process has to be provided, enabling a crossdomain data access. Sharing domain knowledge across all relevant production domains could open completely new opportunities for all process members by closing the gap between them. In this way, highly automated but flexible production systems could be enabled by providing all relevant information in real time to all active entities of the process [4]. Therefore, a coherent technical, syntactical, and semantical interoperability of interaction based on communication is required [5].

To reach horizontal and vertical integration of production components, a common understanding of this process is crucial. For this reason, the Reference Architecture Model for Industry 4.0 has been postulated, called RAMI 4.0. Formalized by the German standardization organization DIN, RAMI 4.0 integrates elements of Industry 4.0 into a three-dimensional layer's model, giving formal definitions of their vertical and horizontal integration and product life-cycle engineering $[6,7]$.

Furthermore, the introduction of Industry 4.0 will be a long transformation process, which will concern wide parts of production systems. At the same time, risks of the change of running systems have to be kept minimal, so that companies still stay competitive. Therefore, the digital transformation of production systems requires a coherent migration path. One eminent approach in [8] delivers 
Fig. 1 RAMI 4.0 following [6]

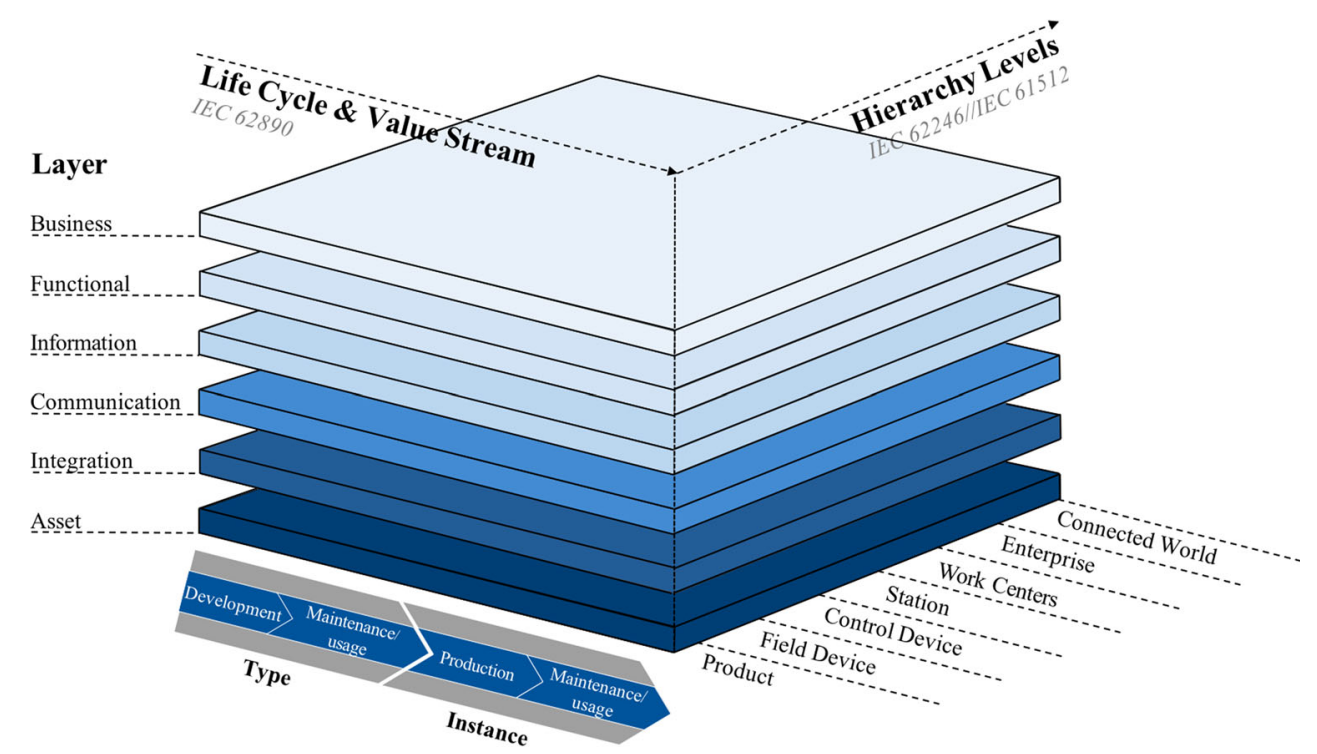

towards this objective, describing possible stages of the transformation of legacy production systems towards smart factories in the sense of Industry 4.0.

The novelty of this study is that an exemplary production development way towards Industry 4.0 is proposed, based on the given approaches of RAMI 4.0, migration path according to [8], and existing automation technologies. The unique highlight is thereby that the study unites technological and economic view on the development process of legacy production systems and provides a concrete implementation example. Past studies in this field mostly miss one of these essential views. In [7], a concept of an Industry 4.0 compatible control device is developed, focusing on its technological suitability, but disregarding its economic profitability for the enterprise. The studies of $[1,2]$ and [9] have a similar deficit, presenting a convincing attempt towards an Industry 4.0 component using existing technologies, but not bringing this in the context of the enterprise development. In [10], the future development direction of Industry 4.0 is analyzed, leaving behind the analysis of enabling technologies. The study of [11] also focuses only on sustainable development of enterprises under Industry 4.0 conditions without analyzing its technological development. Therefore, the concept of this study pursues the objective of uniting all relevant perspectives, including formal standards by using RAMI 4.0 , economical interest of production companies by the migration path and a possible implementation concept by available technologies. Accordingly, a demonstration project of the developed framework is considered to evaluate the feasibility and applicability of the proposed approach.

Therefore, this paper is structured as follows. Section 2 provides a detailed overview about theoretical basics, namely the migration path and RAMI 4.0, and reveals their potential for application to Industry 4.0. Section 3 proposes an exemplary production process evolution concept and discusses underlying automation technologies. An exemplary implementation of the proposed concept, based on a research project from the field of technical fluid maintenance of machine tools, is detailed in Section 4. Finally, the study concludes with a summary and a vision for future work in Section 5.

\section{Theoretical basics}

\subsection{Reference architecture model Industry 4.0}

Many different domains of interest come together in the discussion about the Industry 4.0 development. Factory and process automation approaches, communication and automation technologies, and production standards need to be connected with each other properly, enabling a coherent technical, syntactical, and semantical interoperability. Hence, there is no doubt that a common understanding reference model is required.

Presented at the Hannover Fair in 2015, (shown in Fig. 1) is currently one of the most significant results reported within the context of Industry 4.0 research. It provides a formal framework for Industry 4.0 components, where its essential characteristics for manufacturing systems can be adapted. The model contains three axes, described as follows [6]:

1. The Architecture axis ("Layers"), with six layers giving information about the IT structure of a production system and the asset role in there;

2. The "Life Cycle and Value Stream" axis, representing the lifetime of an asset and its value-added process, based on IEC 62890; 
3) The "Hierarchy Levels" axis, giving an overview about hierarchical levels of a manufacturing system, based on the DIN EN 62264-1 and DIN EN 61512-1 standards.

From an automation technology sight, the great challenge of adopting RAMI 4.0 is in the development of tangible solutions that support the functionalities of each layer and interaction between them. Therefore, this work focuses on the vertical Architecture axis.

At this axis, the Asset layer represents the physical world, i.e., the asset that really exists (machines, machines' parts, axes etc.) and a connection of the asset to its virtual representation in the higher levels.

In the Integration level, a transition from the physical world to the information world occurs. Hence, this layer contains properties and process-related functions that make the asset usable for its intended purpose, including humanmachine interfaces (HMI) and descriptions of technical elements. Each relevant event in the real world triggers an event in the virtual world by being reported to the Integration level.

The Communication layer contains a standardized Industry 4.0 compliant communication using a uniform data format. In other words, it describes the connection of the asset to information and functions of other assets.

The Information layer includes descriptions of all the data used, generated, or modified by the technical functionality of the asset. On this level, consistent integration of different data, its persisting and consistent integration occur.

The Functional layer contains formal, digital description of technical functions of the asset with regard to its role in the production system, providing a platform for horizontal integration of different functions of all assets.
Finally, yet importantly, the Business layer represents higher strategic enterprise view. This includes orchestration of services in the Functional layer, ensuring the integrity of functions in the value-added chain, mapping business models, and the resulting business processes, etc. However, the role of the Business layer is not related to specific solutions such as Enterprise Resource Planning (ERP), which are located in the Functional layer instead [6]

\subsection{Industry 4.0 development path}

Nowadays, production system owners are facing the challenge of adopting Industry 4.0. There is no doubt that it will be a long-lasting process, connected with fundamental changes in almost all parts of an enterprise. Planning and implementation of these changes have to be designed in a way that they continuously have a positive effect on profitability, growth, and efficiency of the manufacturing system and the whole enterprise. The advantage of digital transformation has to be transparent at every moment; otherwise, the entrepreneurs will not support it. Hence, a coherent migration path is required, providing production system owners a guideline for adopting the fundamentals of Industry 4.0 in their enterprises. In [8], one eminent research approach to this objective is provided. This path contains of six sequential development steps, which describe corresponding Industry 4.0 maturity of an enterprise and its estimated business add-value (Fig. 2). It is crucial that the competencies build up gradually, so that each following step is based on the previous one.

The first step Computerization describes an isolated use of information technologies (IT) in an enterprise, for instance, use of a milling machine with computerized
Fig. 2 Industry 4.0 development path following [8]

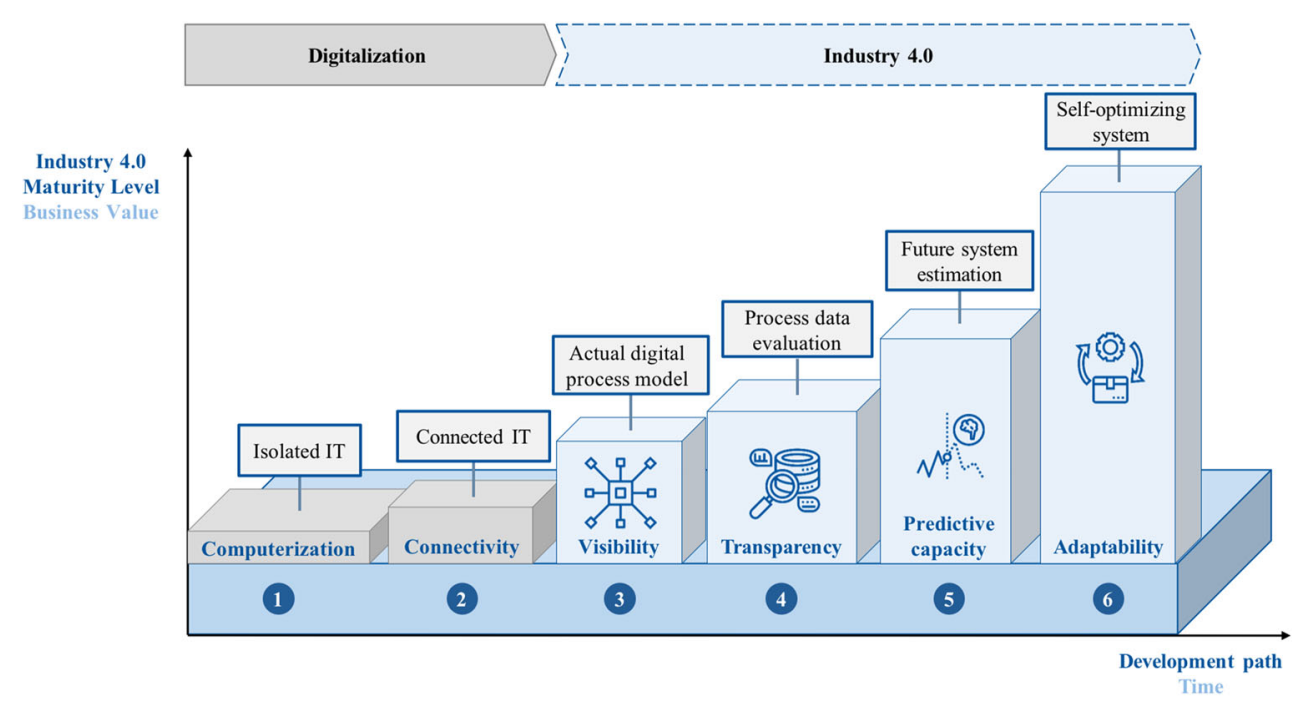


numerical control (CNC). This allows accelerating and improving of easy repetitive activities, so that the main benefit of this step is a higher efficient and less error-prone production. Nowadays, this step is almost accomplished in most German enterprises.

The next step, Connectivity, represents a connected use of IT in the enterprise. Used technologies are able to communicate with each other and first APIs from operational technologies (OT) to business IT are implemented, so that an approximate reflection of core business processes takes place. In this step, for example, handling robots in a production line can communicate with each other and the result of their work is automatically reported to a Manufacturing Execution System (MES). Both steps Computerization and Connectivity are united under the term Digitalization, providing fundamentals for Industry 4.0 while not being its part.

The following step, Visibility, represents a starting point for the Industry 4.0. In this step, an actual digital model of the enterprise is real-time available, providing relevant information about production system's current state. This approach can be defined as a kind of Digital Shadow of the enterprise. By the means of Digital Shadow, strategical systems such as Product Lifecycle Management (PLM) can be integrated properly, making the current production state visible for enterprise managers.

In the Transparency step, already visible production data are aggregated and their detailed analysis is performed, so that system elements' relations can be identified. Data analytics methods such as artificial intelligence (AI) enable such processing of production data. For example, image recognition methods based on $\mathrm{AI}$ can be used to detect surface errors of a workpiece in terms of quality control. Using this errors' data combined with the frequency data of the working machine, dangerous frequencies can be found and further avoided. Hence, a correct estimation of system behavior allows a targeted influence on it in terms of condition monitoring.

In the following step, building of possible future system behavior scenarios based on the analyzed past data is possible. In other words, Predictive capacity is given, so that approaching situations can be anticipated and a responsible manager can timely take relevant decisions and preventive steps.

The difference of the final step of the development path, Adaptability, to the previous step Predictive capacity is the possibility of an autonomous system reaction to a current production state change. In other words, a production system is self-optimizing and can take certain decisions on its own. The automation level is thereby based on decision complexity and cost-benefit relation. In summary, the purpose of the final step of the development path is achieved if the Digital Shadow data can be used for autonomous control in real-time, bringing the enterprise a higher benefit [8].

\section{Production process evolution concept}

According to both theoretical approaches from Chapter 2, a concept of production process evolution is defined, which considers both business value and IT infrastructure of an enterprise. This concept can be applied to derive processspecific implementation steps towards Industry 4.0 based on existing automation technologies.

Figure 3 shows a matrix of defined production process evolution. Its horizontal axis represents the six layers of RAMI 4.0. The vertical axis shows the Industry 4.0 maturity steps according to [8]. In the cells of the built matrix, information and communication technologies are accommodated, which are required to provide the related
Fig. 3 Concept of production process evolution based on the Industry 4.0 maturity steps [8] and RAMI 4.0 [6]

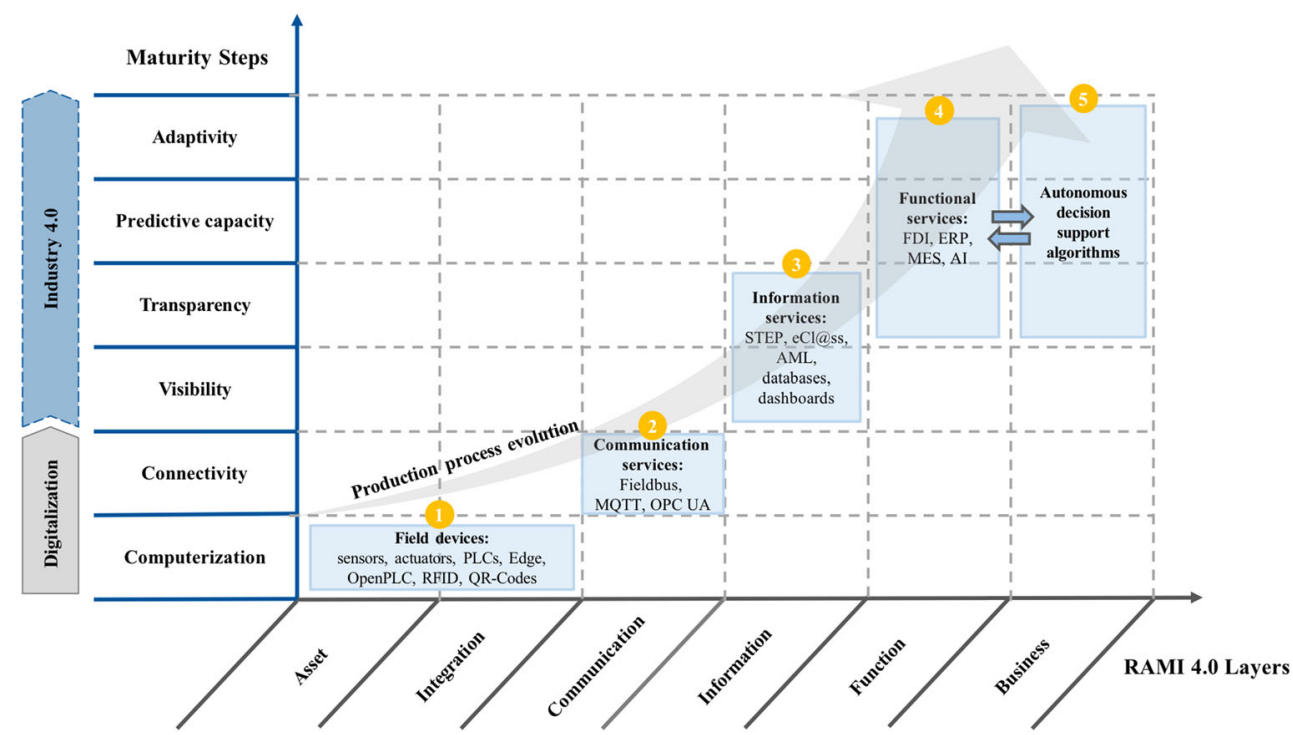


RAMI 4.0 layers' functionality and achieve the value-add of related maturity steps.

Therefore, this paper considers production process evolution towards Industry 4.0 as a sequential process based on a simultaneous development of IT infrastructure and business maturity of an enterprise by implementing "stage-seasoned" automation technologies. These stages are detailed as follows:

\section{Field devices}

At the starting point of the production process evolution, hardware components such as machines, sensors, actuators, and controllers (PLCs) need to be installed properly. In this stage, controllers are mainly responsible for operation management at the machine: for processing sensor data, triggering actuators, and changing machine's state. Edge devices can also be implemented to support PLC functionality and further operation management. Moreover, relevant devices must have a corresponding unique virtual representation in terms of identification. For this, automatic identification and data capture (AIDC) technologies can be used. They include radiofrequency identification (RFID), bar codes, magnetic stripes, etc. An overview about their properties is given in [12], for example. Thus, this starting stage unites the layers Asset and Integration and the first maturity step Computerization.

\section{Communication services}

In the following, installed field devices need to be connected with each other and with user applications to enable continuous data exchange. Thereby, this stage provides communication services (protocols) to meet the needs of the following stages. These protocols must be defined in a technology-independent way to achieve interconnectivity between different field networks and services. Actual data exchange mechanisms in the industrial sector are Fieldbus protocols such as CAN, Modbus, or Profibus and machineto-machine communication mechanisms such as Open Platform Communications Unified Architecture (OPC UA) and Message Queuing Telemetry Transport (MQTT) [7, 13]. As a basic research guideline, [14] recommends OPC UA as the main Industry 4.0 approach due to its superior, stable, and standardized performance. Many authors, such as [13, 15-18], approve this recommendation with their experience. However, OPC UA is complex architecture; therefore, in some ordinary use cases with a few production process members, it could be more reasonable to use Fieldbus or MQTT protocols (see Chapter 4).

\section{Information services}

In this stage, a global data management system needs to be established. This system has to include not only an actual digital representation of the production system, its visualization and data storage, but also continuous filtering and consistent integration of relevant data, thus uniting the Visibility and Transparency stages. One of the main challenges is thereby that the data comes from a variety of engineering tools in different formats; therefore, stage-seasoned technologies have to allow mapping and integration of this data. For this purpose, the following methods are promising: Standard for the Exchange of Product model data (STEP) [13, 19], Systems Modelling Language (SysML) [13], Automation Modelling Language (AML) [1, 13, 20, 21], Electronic Device Description (EDD), classification standard eCl@ss and Field Device Tool (FDT) [13, 14]. For data storage and visualization, different databases and dashboards can be used.

\section{Functional services}

After establishing a coherent information management system, a runtime environment for system applications and its technical functionality has to be provided. In other words, filtered relevant data from different tools and sources enable estimation and anticipation of future system conditions in this stage. According to [14], Field Device Integration (FDI) is a recommended technology to integrate informational and functional services. For system state estimation, methods of artificial intelligence can be implemented. Based on their results, operation management systems such as MES or ERP can be used for strategic decisions for both technical (e.g., predictive maintenance) and business (sales volumes, etc.) sights.

\section{Autonomous decision support algorithms}

This last stage communicates closely with the previous one. According to [6], the functionality of the Business layer is not associated to specific solutions: They are all assigned to the Functional layer. Therefore, implementation technologies are not explicitly listed for this final stage of production process evolution. Its focus is on the development of decision support algorithms, which can optimize system behavior from both the functional and the commercial views. Overall, this stage represents an orchestration of services in the previous stages, which is autonomous and self-optimizing to a reasonable extent.

\section{Implementation example}

In machine tools, technical fluids in the form of cutting fluids (CFs) are used for cooling and lubricating between tool and workpiece and for removing chips. The $\mathrm{CF}$ concentration must be adjusted depending on different 
machine and process parameters and must be checked regularly. The use of the correctly adjusted CF contributes to better dimensional accuracy and surface quality of the machined workpieces and reduces tool wear. At the same time, improperly treated CF can become a hazardous substance that can cause illness and release toxic substances [22]. Nevertheless, the maintenance and care of the CF are often neglected due to non-transparency or lack of process documentation.

In the MoTeF 4.0 project, the FLACO-Geräte GmbH and the WZL of RWTH Aachen in cooperation are developing a mobile fluid dosing system with connection to digital production control systems for an automatic, demandoriented, and documented filling of technical fluids on machine tools. The newly developed fluid dosing system detects the machine tool, measures the level of the CF in the tank, processes sensor data on the condition of the fluid, and refills according to the target parameters of the production control system. The test parameters as well as the delivered quantity and concentration of the CF per machine are logged, whereby the consumption of each machine tool is available as historical data. The overall structure of the proposed system is shown in Fig. 4.

The supposed development of the novel fluid dosing system in the MoTeF 4.0 project is an example for the application of the concept presented in this paper. An already existing manual device for the dosing of cutting fluids will be refined regarding to its application in the context of Industry 4.0. The existing device is essentially a mixing station where all the parameters required for dosing the CF must be entered manually. This mixing station is placed on a small carriage, which can be driven directly to the machine to be filled [23]. There is no automated parameter acquisition, data storage, or external connection.
All these features are added through different stages of production process evolution, presented in Fig. 5.

\section{Field devices}

In the tank of the machine, sensors are integrated to automatically measure the concentration of the cooling lubricant, its $\mathrm{pH}$ value, nitrite and nitrate contents, and the filling level of $\mathrm{CF}$ in the tank. All sensors are connected to a PLC at the machine. For machine identification, a RFID transmitter is attached to the machine and a proper reader to the carriage. On the carriage, another PLC with a touch display is installed, which calculates the amount and concentration of the CF to be refilled. The PLC also controls the pumps of the mixing station and checks the right amount of filled liquid through flow sensors. A machine operator can identify himself by using the display and subsequently start the refilling. Each PLC is connected to an Edge device (Revolution Pi), which serves as a WLAN access point and enables further processing of the acquired data.

\section{Communication services}

Between the PLCs and their related Edge devices, a PLC specific connection is established. The Edge devices are connected to the local WLAN. An MQTT Broker is implemented on each Edge device, providing gathered data to all interested process members, e.g., to the database (s. next stage). MQTT as a communication protocol is chosen due to its simple implementation and possibility to share data with many different process members.

\section{Information services}

In order to ensure the comprehensibility of the data, the following structure is implemented. A database (InfluxDB) located at a local enterprise's server subscribes to the

Fig. 4 Structure of the MoTeF 4.0 project

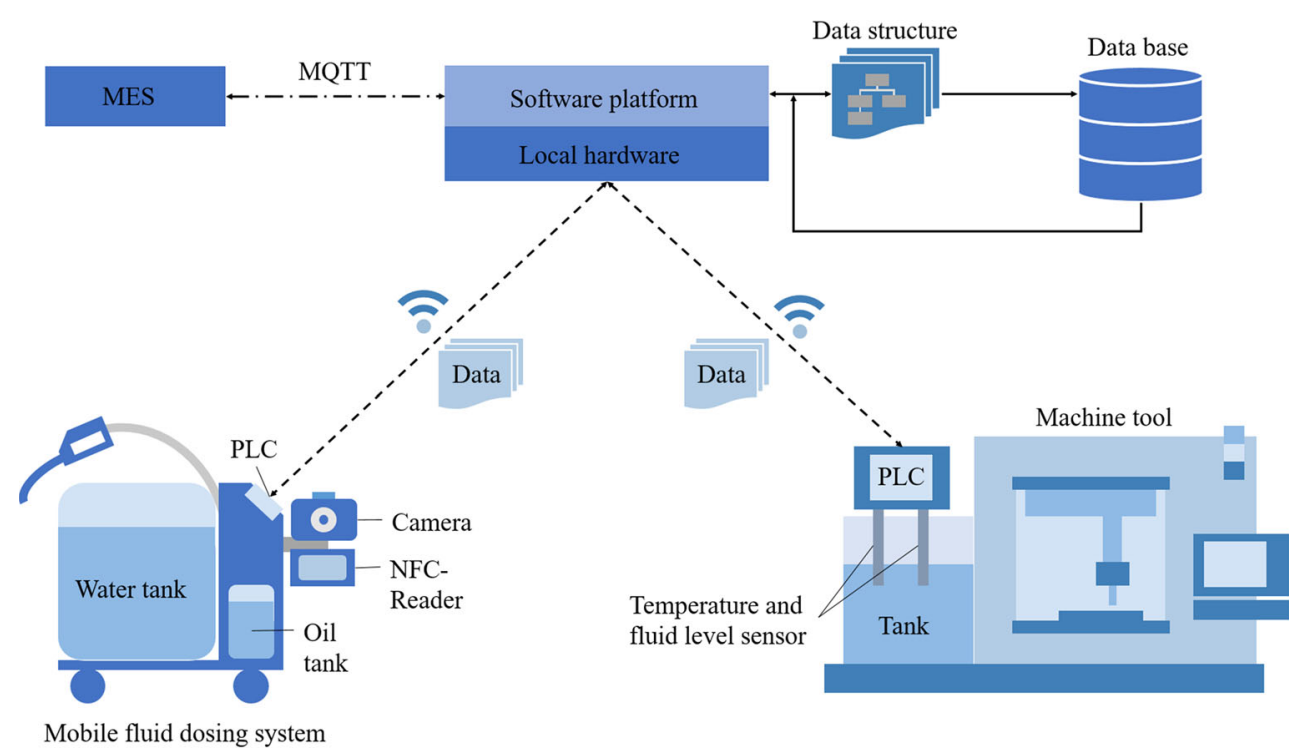


Fig. 5 Stages of production process evolution for the $\mathrm{MoTeF}$ 4.0 project

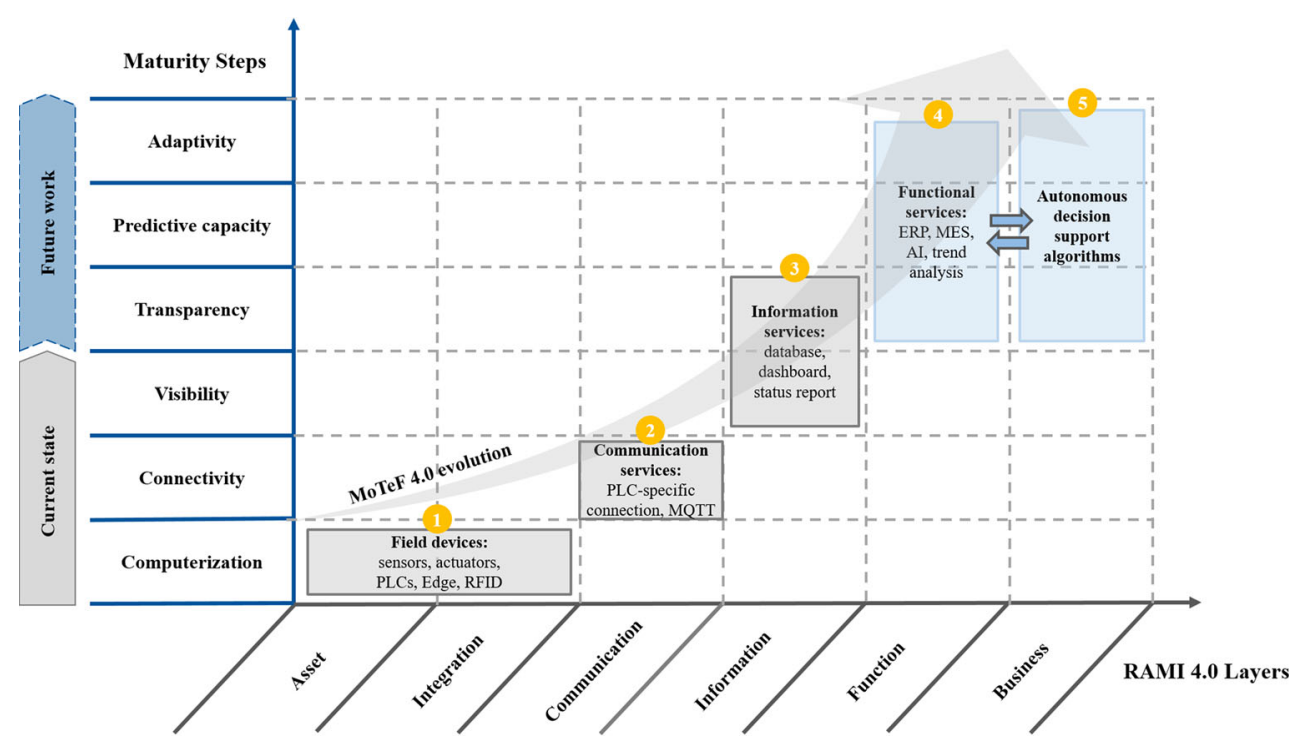

MQTT-Brokers and stores all incoming data: the concentration of cooling lubricant, its $\mathrm{pH}$ value, nitrite and nitrate content, machine ID and filling level, etc. Located at the same server, a suitable dashboard (build with Grafana) is connected to the database and visualizes this data. The visualization allows the operator at the shop floor and the higher management to see all relevant information about actual fluid system state on their screens. Additionally, a status report is regularly sent to a selected repository and stored as history data for further data processing. Given the fact that the process data is homogenous, coming from allied engineering tools, no further integration tools are implemented.

As shown in Fig. 5, this stage represents the current state of the MoTeF 4.0 project. Described field devices, communication, and information services are implemented already, providing connectivity and visibility of the CF dosing process.

\section{Functional services}

MES and ERP systems can subscribe to the MQTT brokers of the Edge devices or access the database to receive all information about the $\mathrm{CF}$ handling process. This information can be used by management for strategical decisions on production planning, e.g., about further $\mathrm{CF}$ supply amounts. Moreover, the PLC on the carriage gets the information from the MES about the amount and the concentration of $\mathrm{CF}$ to be filled in the related machine, so that the field operator is provided by correct working instructions. Furthermore, AI methods are considered to predict future system behavior and deliver action recommendations. Based on the stored history data, the date of a possible machine breakdown because of CF lack in the tank can be predicted by a trend analysis, enabling predictive maintenance. Taking countermeasures early allows enterprises to avoid downtime costs, for instance.

\section{Autonomous decision support algorithms}

Decision support algorithms in the MES and on the PLC of the carriage help to estimate CF consumption and maintenance, which enables automatic, demand-oriented, and documented filling of technical fluids. The field operator takes the carriage, walks to a machine, and has to start the filling without entering any parameters or machine identification codes. He receives all instructions from the implemented systems. Possible errors and their resulting costs are thus minimized. Therefore, the system is autonomous and self-optimizing to a reasonable extent and the goal of the project is achieved.

\section{Conclusion}

By reference to the RAMI 4.0 and the Industry 4.0 maturity path according to [8], a concept of production process evolution based on existing automation technologies is presented in this study. The characteristics and functionalities of each concept stage are elaborately discussed and the technology selection and assignment to respective stages are justified. To evaluate the feasibility and applicability of the proposed approach, an exemplary implementation project from the field of technical fluid maintenance is detailed. Overall, this 
study aimed to provide a theoretical sequential guideline for production process development towards Industry 4.0, considering both IT infrastructure and business value-add of an enterprise.

In future work, the described application project will be further developed, so that validation and further ascertainment of the proposed concept take place. Further maturity steps will be implemented. Automation technologies, which are best suitable for this purpose, will be further investigated and examined for their Industry 4.0 suitability. Subsequently, their general validity for other applications will be discussed, including evaluation of the effects of presumptions on the proposed method. Another goal of the future work is to evaluate the benefits of the named technologies in terms of their mutual use within a global production system framework.

Acknowledgements Funded by the Deutsche Forschungsgemeinschaft (DFG, German Research Foundation) under Germany's Excellence Strategy - EXC-2023 Internet of Production - 390621612; also funded by the German Federal Ministry for Economic Affairs and Energy within the MoTeF 4.0 project.

Author contribution C. Brecher and A. Müller elaborated the concept of the study. A Müller and Y. Dassen implemented the example from the field of cutting fluids. S. Storms participated in the design of the study and its coordination and helped to draft the manuscript. All the authors read and approved the final manuscript.

Funding Open Access funding enabled and organized by Projekt DEAL. Funded by the Deutsche Forschungsgemeinschaft (DFG, German Research Foundation) under Germany's Excellence Strategy - EXC-2023 Internet of Production - 390621612; also funded by the German Federal Ministry for Economic Affairs and Energy within the MoTeF 4.0 project.

\section{Declarations}

Consent to participate The authors agree to participate in the submission and publication.

Consent for publication The authors agree to publish the submitted paper according to the rules of the journal.

Competing interests The authors declare no competing interests.

\section{References}

1. Luder A, Schleipen M, Schmidt N, Pfrommer J, Henssen R (2017) One step towards an Industry 4.0 component. In: 2017 13th IEEE Conference on Automation Science and Engineering (CASE), Xi'an, pp 1268-1273

2. Tantik E, Anderl R (2017) Integrated Data Model and Structure for the Asset Administration Shell in Industrie 4.0. Procedia CIRP 60:86-91. https://doi.org/10.1016/j.procir.2017.01.048
3. Bundesministerium für Wirtschaft und Energie (BMWi) (2020) Leitbild 2030 für Industrie 4.0 - Digitale Ökosysteme global gestalten, monthly report of the Industrie 4.0 Working Group, Oct. 2019. Accessed: Mar. 20 2020. [Online]. Available: https://www.bmwi.de/Redaktion/DE/Downloads/Monatsbericht/ Monatsbericht-Themen/2019-10-leitbild-2030-fuer-industrie-40. pdf?__blob=publicationFile \& v $=6$

4. Bangemann T, Bauer C, Bedenbender H, Diesner M, Epple U, Elmas F, Friedrich J, Goldschmidt T, Göbe F., Gruner S, Hankel M, Hesselmann K, Hüttemann G., Kehl H, Löwen U., Pfrommer J, Schleipen M, Schlish B, Usländer T., Werterkamp C, Winter A, Wollschläger M. (2016) Industrie 4.0 - Technical Assets: basic terminology concepts life cycles and administration models: Status report

5. Ali Shah PA, Habib M, Sajjad T, Umar M, Babar M (2017) Applications and challenges faced by internet of things - a survey. In: Ferreira J., Alam M. (eds) Lecture notes of the institute for computer sciences, social informatics and telecommunications engineering, future intelligent vehicular technologies. Springer International Publishing, Cham, pp 182-188

6. (2016). Reference Architecture Model Industry 4.0 (RAMI 4.0), DIN Spec 91345

7. de Melo PFS, Godoy EP (2019) Controller interface for industry 4.0 based on RAMI 4.0 and OPC UA. In: 2019 II Workshop on Metrology for Industry 4.0 and IoT (MetroInd4.0 \& IoT), Naples, Italy, pp 229-234

8. Schuh G, Anderl R, Gausemeier J, ten Hompel M, Wahlster W (2017) Industrie 4.0 Maturity Index. Die digitale Transformation von Unternehmen gestalten, acatech STUDIE, München

9. Contreras JD, Pastrana JDD, Melo JIG (2017) Developing of industry 4.0 applications. International Journal of Online Engineering (iJOE)

10. Cheng G, Liu L, Qiang X, Ye L (2016) Industry 4.0 development and application of intelligent manufacturing. In: 2016 International conference on information system and artificial intelligence

11. Skorobogatova N (2019) Sustainable development of an enterprise under industry 4.0 conditions. In: 2019 International conference on creative business for smart and sustainable growth (CREBUS)

12. Helmus M, et al. (eds) (2009) RFID in der Baulogistik: Forschungsbericht zum Projekt. Integriertes Wertschöpfungsmodell mit RFID in der Bau- und Immobilienwirtschaft, 1st edn. Vieweg, Wiesbaden. Accessed: Feb. 20 2020

13. Ye X, Hong SH (2018) An automationML/OPC UA-based industry 4.0 solution for a manufacturing system. In: 2018 IEEE 23rd International Conference on Emerging Technologies and Factory Automation (ETFA), Turin, pp 543-550

14. BITKOM e.V., VDMA e.V., ZVEI e.V. (2015) Umsetzungsstrategie Industrie 4.0: Results Report of the platform Industrie 4.0, pp. 40-45. Accessed: 20.03.20. [Online]. Available: https://www.bitkom.org/sites/default/files/file/import/ 150410-Umsetzungsstrategie-0.pdf

15. Schleipen M, Gilani S.-S., Bischoff T, Pfrommer J (2016) OPC UA \& Industrie 4.0 - Enabling Technology with High Diversity and Variability. Procedia CIRP 57:315-320. https://doi.org/10.1016/j.procir.2016.11.055

16. Derhamy H, Ronnholm J, Delsing J, Eliasson J, van Deventer J (2017) Protocol interoperability of OPC UA in service oriented architectures. In: 2017 IEEE 15th International conference on industrial informatics (INDIN), Emden, pp 44-50

17. Gruner S, Pfrommer J, Palm F (2016) RESTful industrial communication with OPC UA. IEEE Trans Ind Inf 12(5):18321841. https://doi.org/10.1109/TII.2016.2530404, 
18. Luo $\mathrm{Z}$ et al (2017) OPC UA-based smart manufacturing: system architecture, implementation, and execution. In: 2017 5th International conference on enterprise systems (ES), Beijing, pp 281-286

19. Wei Q, Duan G, Cai J, Zhou G (2009) Mapping technique of STEP data model in relational database based on data storage

20. Lüder A, Schmidt N (2017) AutomationML in a nutshell. In: Vogel-Heuser B, Bauernhansl T, ten Hompel M (eds) Handbuch Industrie 4.0 Bd.2. Springer, Berlin, pp 213-258

21. Vogel-Heuser B, Bauernhansl T, ten Hompel M (eds) (2017) Handbuch Industrie 4.0 Bd.2. Springer, Berlin
22. (1981) Pflege von Kühlschmierstoffen, VDI-3397 Page 1

23. (2020) FLACO-Geräte GmbH, FLACO KSS 200/60pro - mobiles Misch- \& Befüllsystem für Kühlschmiermittel. [Online] Available: https://www.flaco.de/fileadmin/flaco/media/Downloads/ FIS-PDFs/FLACO_KSS_FLYER_200-60_PRO_DOC.pdf.

Accessed: 03/24/2020

Publisher's note Springer Nature remains neutral with regard to jurisdictional claims in published maps and institutional affiliations. 